\title{
Strong Localization of Photons in Certain Disordered Dielectric Superlattices
}

\author{
Sajeev John \\ Department of Physics, Princeton University, Princeton, New Jersey 08544 \\ (Received 5 March 1987)
}

\begin{abstract}
A new mechanism for strong Anderson localization of photons in carefully prepared disordered dielectric superlattices with an everywhere real positive dielectric constant is described. In three dimensions, two photon mobility edges separate high- and low-frequency extended states from an intermediatefrequency pseudogap of localized states arising from remnant geometric Bragg resonances. Experimentally observable consequences are discussed.

PACS numbers: 71.55.Jv, 42.20.-y, 78.30.Ly
\end{abstract}

Since the pioneering work of Anderson ${ }^{1}$ and Mott $^{2}$ considerable effort has been placed on the observation of electronic localization in disordered solids. An unequivocal test of the scaling theory of localization, ${ }^{3}$ however, has been hampered by the nearly inescapable presence of electron-electron interactions and electron-phonon scattering in real materials. Recently it has been suggest$\mathrm{ed}^{4,5}$ that the Anderson-localization transition may be observed for electromagnetic waves propagating in strongly scattering dielectric structures. Optical systems provide an ideal experimental realization of a single noninteracting excitation in a static random potential. In dense random systems with a high dielectric mismatch between scattering structures and background, it was suggested ${ }^{4}$ that an intermediate-frequency window of localized states separates low-frequency extended states exhibiting Rayleigh scattering from high-frequency extended states in which propagation is described by geometric optics. More detailed treatments ${ }^{6-8}$ including the vector nature of the photon and the possibility of single-scattering Mie resonances have provided support to this basic physical picture. From an experimental point of view, however, the fundamental challenge lies in the preparation of disordered dielectric microstructures in which the predicted conditions for strong localization of photons may be achieved. The basic mechanism for renormalization of transport coefficients and localization is the coherent backscattering of light. The existence of this precursor to the Anderson transition has been verified in a variety of weak-scattering systems. ${ }^{9-12}$ The observed dependence of the polarization content and backscattering line shape on sample geometries and adsorption are in good agreement with theory. ${ }^{13-15}$ In all of these systems, however, the elastic mean free path $l$ is at least an order of magnitude longer than the photon wavelength $\lambda$, thereby precluding any mobility-edge behavior for which it is required ${ }^{16}$ that $2 \pi l / \lambda \simeq 1$. Recently Genack ${ }^{17}$ has reported mean free paths $l \lesssim \lambda$ in certain titania microstructures. This unprecedented finding, however, depends sensitively on the large-scale structural arrangement of titania spheres. In these relatively dense random systems, the dielectric spheres are optically con- nected and single-scattering Mie-resonance theories are inapplicable. It is the purpose of this paper to elucidate instead the importance of large-scale geometric resonances in disordered systems. Localization produced by such geometric resonances is the result of a delicate interplay between order and disorder. The most familiar example of such a resonance is the Bragg scattering of an electron in a perfect crystal. The utilization of this mechanism by means of band-gap engineering in superlattices has led to novel applications in electronics. The persistence of this mechanism in disordered systems is evident from the existence of electronic band gaps or pseudogaps in amorphous semiconductors. ${ }^{18}$ On the other hand, the application of this mechanism to photonics has for the most part been restricted to one-dimensional superlattices. Here it is well known that Bragg reflection gives rise to forbidden frequency bands or stop gaps where optical propagation cannot occur and the electromagnetic intensity decays exponentially with distance into the medium. ${ }^{19}$ Recently, Yablonovitch ${ }^{20}$ has suggested that a three-dimensional periodic array of dielectric scatterers possessing such a gap may lead to the inhibition of spontaneous emission. It is my hypothesis that carefully prepared three-dimensional photonic superlattices with moderate disorder may provide the key to the predictable and systematic observation of strong localization of photons in nondissipative materials with an everywhere real positive dielectric constant.

The occurrence of pseudogaps in the photon density of states (DOS) necessitates a reinterpretation of the standard Ioffe-Regel condition. Previously ${ }^{21}$ this condition was derived with use of the approximation of a freephoton density of states and perturbation theory for repeated single scattering from various dielectric spheres. The disorder-averaging procedure, however, leads to a smearing of any macroscopic geometric resonances. If, instead, one treats these geometric resonances exactly prior to the performing of a disorder average, then the wavelength entering the Ioffe-Regel condition must be interpreted as the inverse of the fluctuation in the wave vector from the Bragg plane rather than the free-photon wavelength itself. Since this wavelength diverges at a 
band edge, localization is possible even for weak randomness.

The vector nature of the electromagnetic field has important consequences for the Bragg backscattering mechanism. The existence of two helicity channels for any given photon of wave vector $\mathbf{k}$ makes the realization of band gaps or near gaps in three dimensions more difficult than in the corresponding electronic problem and leads to stronger restrictions on the minimum required fluctuation in the amplitude of the dielectric constant. We consider the wave equation for the electric field amplitude $\mathbf{E}$ for radiation of frequency $\omega$ in an isotropic disordered medium,

$$
-\nabla^{2} \mathbf{E}+\nabla(\nabla \cdot \mathbf{E})-\frac{\omega^{2}}{c^{2}} \epsilon_{\text {fluct }}(\mathbf{x}) \mathbf{E}=\epsilon_{0} \frac{\omega^{2}}{c^{2}} \mathbf{E} .
$$

Here $\epsilon_{0}$ is the mean value of the dielectric constant and the fluctuating part $\epsilon_{\text {fluct }}=\epsilon_{1}(x)+V(x)$ has two real parts, the first which describes a Bravais superlattice,

$$
\epsilon_{1}(x)=\epsilon_{1} \sum_{\mathbf{G}} U_{\mathbf{G}} e^{i \mathbf{G} \cdot \mathbf{x}}
$$

and the second which is random and satisfies the statistics $\langle V(x)\rangle_{\text {ens }}=0$ and $\langle V(x) V(y)\rangle \equiv B(x-y)$. The summation in (1b) runs over the reciprocal lattice $\{\mathbf{G}\}$ which has the inversion symmetry $U_{\mathbf{G}}=U_{-\mathbf{G}}$. An analogous separation of periodic order and disorder is inherent in tight-binding theories of disordered electronic systems. The effect of the periodic modulation on the photon spectrum may be estimated within a nearly-free-photon approximation. For right- and left-hand circularly polarized photons of wave vector $\mathbf{k}$ in the vicinity of a single Bragg plane the effects of scattering into a degenerate state of wave vector $\mathbf{k}-\mathbf{G}$ are described by first-order degenerate perturbation theory. Unlike scalar electrons which may be described by a second-order determinant, ${ }^{22}$ there is an additional degeneracy in the photon helicity state as well as the possibility of helicity-flip scattering. The resulting eigenvalue condition is

$$
\operatorname{det}\left|\begin{array}{cccc}
\alpha(\mathbf{k}) & 0 & 1-f & f \\
0 & \alpha(\mathbf{k}) & f & 1-f \\
1-f & f & \alpha(\mathbf{k}-\mathbf{G}) & 0 \\
f & 1-f & 0 & \alpha(\mathbf{k}-\mathbf{G})
\end{array}\right|=0
$$

where

$$
\alpha(\mathbf{k}) \equiv\left[k^{2}-\epsilon_{0}\left(\omega^{2} / c^{2}\right)\right] /\left(\epsilon_{1} U_{\mathbf{G}} \omega^{2} / c^{2}\right)
$$

and $f=(1-\cos \theta) / 2$ is the helicity-flip amplitude for scattering from $\mathbf{k}$ to $\mathbf{k}-\mathbf{G}$ by an angle $\theta$. In general, $\cos \theta=(k-\hat{\mathbf{k}} \cdot \mathbf{G}) /|\mathbf{k}-\mathbf{G}|$, whereas for $\mathbf{k}$ lying on the Bragg plane defined by the reciprocal-lattice vector $\mathbf{G}$, the helicity-flip amplitude $f$ reduces to $G^{2} / 4 k^{2}$. Along this Bragg plane, the solutions of the eigenvalue equation (2) are

$$
\omega / c=k\left(\epsilon_{0} \pm \epsilon_{1} U_{\mathbf{G}}\right)^{-1 / 2}
$$

and

$$
\omega / c=k\left(\epsilon_{0} \pm \epsilon_{1} U_{\mathbf{G}}\left|1-G^{2} / 2 k^{2}\right|\right)^{-1 / 2} .
$$

The associated photon dispersion relations are depicted in Fig. 1. At the center of the Bragg plane $\mathbf{k}=\mathbf{G} / 2$ the two branches (3a) and (3b) are degenerate, whereas if the point $k=G / \sqrt{2}$ is accessible on the Brillouin-zone surface it is apparent from (3b) that the gap is closed as a result of the additional photon helicity channel. If we set $U_{\mathbf{G}}=1$, it is also apparent from these solutions that the lowest-frequency state of the upper branch ( - sign) occurs at $\mathbf{k}=\mathbf{G} / 2$ provided that $\epsilon_{1} / \epsilon_{0} \leq \frac{1}{3}$, whereas for higher-amplitude periodic modulations $\left(\epsilon_{1} / \epsilon_{0}>\frac{1}{3}\right)$ the corresponding minimum occurs along a circle defined by the intersection of the Bragg plane with the sphere $k^{2}=G^{2} /\left(1+\epsilon_{0} / \epsilon_{1}\right)$. The frequency at the bottom of the upper branch then becomes

$$
\omega / c=\left(2 \epsilon_{1}\right)^{1 / 2} G /\left(\epsilon_{0}+\epsilon_{1}\right), \epsilon_{1} / \epsilon_{0}>\frac{1}{3} .
$$

As an illustration we consider an fcc Bravais lattice for which the first Brillouin zone is shown in Fig. 1. A severe depression in the photon DOS occurs for those frequencies $\omega$ which remain in the spectral gap between the upper and lower branches of Eqs. (3a) and (3b) as the wave vector $\mathbf{k}$ is allowed to span the surface of the Brillouin zone. A more complete treatment would require special attention to those points along the zone surface where two or more Bragg planes intersect. The eigenvalue equation corresponding to (2) would involve
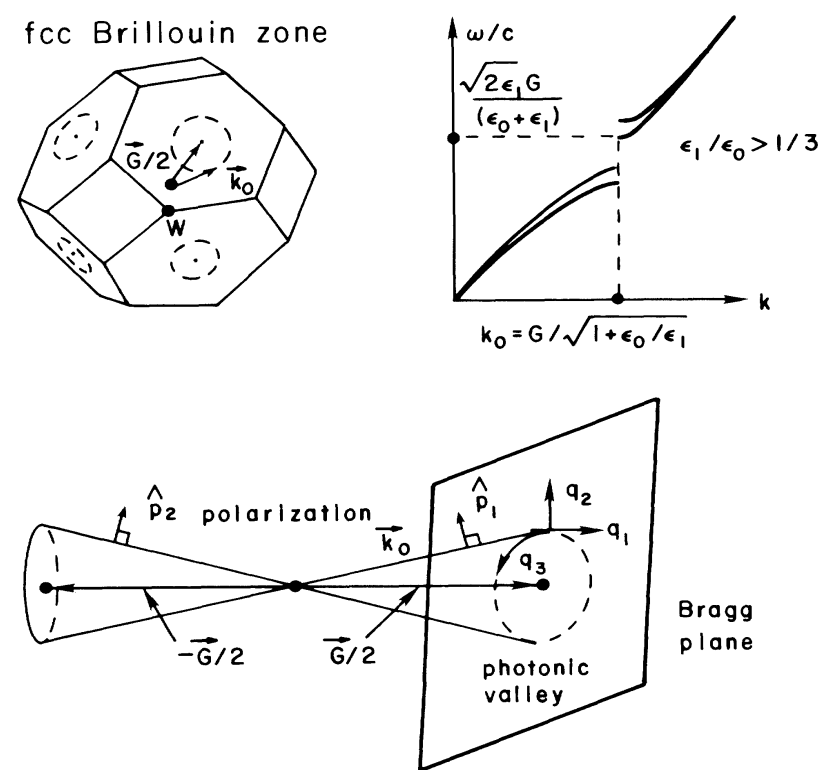

FIG. 1. Creation of a circular photonic valley near the band edge by the Bragg resonance of $p$-polarized light of wave vector $\mathbf{k}_{0}+\mathbf{q}$ in a high dielectric contrast $\epsilon_{1} / \epsilon_{0}$ fcc superlattice. $q_{1}$, $q_{2}$, and $q_{3}$ are principal axes for photon dispersion. 
sixth- and eighth-order determinants. However, since these higher-degeneracy points constitute a set of considerably lower measure than the bulk of the zone surface, the qualitative aspects of the photon DOS may be deduced from the fourth-order determinant (2). More realistic photon band structure will be discussed elsewhere. Since at the extremal point $W$ of the zone surface depicted in Fig. $1, k^{2}=\frac{5}{12} G^{2}<G^{2} / 2$, the gap between the upper and lower branches of (3b) is not closed. In the vicinity of $W$, the lower branch of (3b) acquires a frequency $\omega / c=(5 / 12)^{1 / 2} G /\left(\epsilon_{0}+\epsilon_{1} / 5\right)^{1 / 2}$. The condition that this frequency lie below the bottom of upper branch is $\epsilon_{1} / \epsilon_{0}>35-20 \sqrt{3} \simeq 0.36$. This latter restriction on the amplitude of the dielectric mismatch is consistent with the initial assumption that $\epsilon_{1} / \epsilon_{0}>\frac{1}{3}$. The reader may verify that if instead we considered $\epsilon_{1} / \epsilon_{0}$ $<\frac{1}{3}$ the gap between the upper and lower branches of (3a) and (3b) would in fact close as $\mathbf{k}$ spans the zone surface for this particular (fcc) example. If we regard the superlattice as consisting of an array of dielectric particles of dielectric constant $\epsilon_{a}$ in a background dielectric $\epsilon_{b}$, then the change of variables $\epsilon_{0}=\left(\epsilon_{a}+\epsilon_{b}\right) / 2$ and $\epsilon_{1}=\left(\epsilon_{a}-\epsilon_{b}\right) / 2$ yields the condition $\epsilon_{a} / \epsilon_{b} \gtrsim 2.13$ for the occurrence of a significant pseudogap structure in the photon DOS. This latter condition is easily accessible in the visible spectrum for $\mathrm{TiO}_{2}, \mathrm{Ge}$, and $\mathrm{Si}$ microstructures. The perturbative introduction of disorder $V(\mathbf{x})$ now gives rise to localized states in the pseodogap region as depicted in Fig. 2. In the vicinity of the critical frequency (4) the phase space available for photon propagation is restricted to a set of narrow symmetry-related cones in $\mathbf{k}$ space analogous to the pockets of electrons near a conduction-band edge well known in semiconductor physics. ${ }^{22}$ Coherent backscattering of light must occur by means of disorder-induced scattering within and between such valleys in phase space. The guiding of photons along particular directions by this remnant of the underlying superlattice geometry provides a powerful mechanism for the strong localization of light.

The dispersion relation for photons just above the spectral gap may be expressed in terms of the small deviation $\mathbf{q}$ of the wave vector $\mathbf{k}$ from the point $\mathbf{k}_{0}$ on the Bragg plane for which $k_{0}^{2}=G^{2} /\left(1+\epsilon_{0} / \epsilon_{1}\right)$ (see Fig. 1). The components of $\mathbf{q}$ along the principal axes $\hat{\mathbf{G}}$ and $\hat{\mathbf{g}}$ are labeled $q_{1}$ and $q_{2}$, respectively. The third component $q_{3}$ tangent to the circle describes degenerate states and

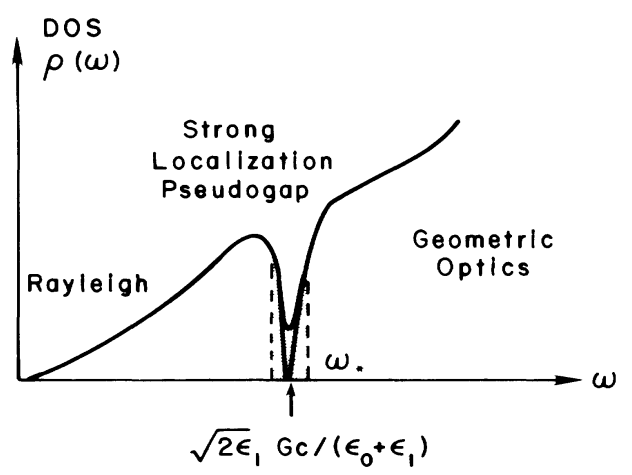

FIG. 2. Photon density of states in a disordered superlattice exhibiting low-frequency Rayleigh scattering and high-frequency geometric-optics extended states separated by a pseudogap of strongly localized photons.

does not enter the dispersion relation at quadratic order of the lowest photon branch $\left(\epsilon_{1} / \epsilon_{0}>\frac{1}{3}\right)$ :

$$
\omega^{2} / c^{2}=E_{C}+A_{1} q_{1}^{2}+A_{2} q_{2}^{2},
$$

where

$$
\begin{aligned}
& E_{C} \equiv 2 k_{0}^{2} /\left(\epsilon_{0}+\epsilon_{1}\right), \\
& A_{1} \equiv\left(2 / \epsilon_{1}\right)\left[\left(\epsilon_{0}^{2}+\epsilon_{1}^{2}\right) /\left(\epsilon_{0}^{2}-\epsilon_{1}^{2}\right)\right],
\end{aligned}
$$

and

$$
A_{2} \equiv 2\left(3-\epsilon_{0} / \epsilon_{1}\right) /\left(\epsilon_{0}+\epsilon_{1}\right) .
$$

The presence of any randomness $V(x)$ leads to a mixing of all nearly degenerate photon branches. Among the contributions to this disorder are frozen phonons, point defects, line defects, and grain boundaries. Shorterwavelength components of the disorder result in intervalley scattering whereas longer-wavelength phonons lead primarily to intravalley scattering. These effects invariably lead to a smearing in wave-vector space of sharp Bragg resonances as well as a filling in of the pseudogap in the photon DOS. I postulate, however, that photons near the band-edge frequency (4) retain certain general features of the dispersion relation (5) and that the phase space available for propagation is accordingly restricted. The dynamics of both intervalley and intravalley scattering are described approximately by an effective Schrödinger equation in wave-vector space,

$$
\left(A_{1} q_{1}^{2}+A_{2} q_{2}^{2}\right) \Psi\left(\mathbf{k}_{v}, \mathbf{q}\right)+\frac{1}{\epsilon_{0}} \frac{\omega^{2}}{c^{2}} \sum_{\text {valleys }} \oint_{\mathbf{k}_{v}^{\prime}} \int d^{2} q^{\prime} \tilde{V}\left(\mathbf{k}, \mathbf{k}^{\prime}\right) \Psi\left(\mathbf{k}_{v}^{\prime}, \mathbf{q}^{\prime}\right)=\left(\frac{\omega^{2}}{c^{2}}-E_{C}\right) \Psi\left(\mathbf{k}_{v}, \mathbf{q}\right)
$$

Here $\mathbf{k}=\mathbf{k}_{v}+\mathbf{q}$ (likewise for primed variables) and $\Psi\left(\mathbf{k}_{t}, \mathbf{q}\right)$ is the $\mathbf{q}$ Fourier transform of an envelope function $\Psi\left(\mathbf{k}_{t}, \mathbf{x}\right)$ which multiplies the carrier-wave electric field eigenvector

$$
\mathbf{E}_{0}\left(\mathbf{k}_{l}, \mathbf{x}\right) \equiv\left[\hat{\mathbf{p}}_{1} e^{i \mathbf{k}_{r} \cdot \mathbf{x}}+\hat{\mathbf{p}}_{2} e^{i\left(\mathbf{k}_{v}-\mathbf{G}\right) \cdot \mathbf{x}}\right] / \sqrt{2}
$$


describing Bragg scattering at the bottom of a valley $v$. The polarization vectors $\hat{\mathbf{p}}_{i}$ lie in the plane of Bragg scattering defined by $\mathbf{k}_{v}$ and $\mathbf{k}_{v}-\mathbf{G}$, and are unit vectors normal to the surface of the wave-vector cone depicted in Fig. 1. Integration over $\mathbf{k}_{v}^{\prime}$ in (6) is along the intersection of this cone with the Bragg plane. $\tilde{V}$ is the matrix element of the random potential $V(x)$ between the actual electric fields (product of carrier wave and envelope) in the two states $\mathbf{k}$ and $\mathbf{k}^{\prime}$. Ensemble-averaged Green's functions for Eq. (6) may be evaluated by standard techniques. ${ }^{23}$ Perturbation theory in $V(x)$ leads to a disorder-induced shift of the band edge to a frequency $\omega^{2} / c^{2}=E_{C}^{\prime}<E_{C}$ and a photon mobility edge which typically occurs between $E_{C}^{\prime}$ and $E_{C}$ (weak randomness). There is also the possibility of nonperturbative contributions in the form of strongly localized photonic band-tail states $^{24}$ analogous to gap solitons ${ }^{20}$ in nonlinear materials.

Photon transport near a mobility edge $\omega_{*}$ may be described according to the scaling theory of localization ${ }^{3,4}$ in which the photon diffusion coefficient

$$
D(L)=(\bar{c} l / 3)\left(l / L+l / \xi_{\text {coh }}\right)
$$

depends on the macroscopic length scale $L$ of the sample. Here $l$ is the photon elastic-scattering mean free path and $\bar{c}$ is an effective velocity given approximately by the speed of light divided by an effective-medium refractive index. Also, the coherence length $\xi_{\text {coh }}$ diverges as $\left|\omega-\omega_{*}\right|^{-1}$ from the extended-state side as $\omega \rightarrow \omega_{*}$. The average time of flight of a photon across a slab of thickness $L$ is given by $\tau(L)=L^{2} / D(L)$. It is apparent that in the critical regime $\left(\xi_{\mathrm{coh}} \gg L\right)$ this time of flight $\tau \sim L^{3}$ rather than the classical diffusion result $\tau \sim L^{2}$. This critical slowing down of the photon due to incipient localization may also be observable in long-time tails in picosecond-pulse propagation. In particular the transmitted pulse exhibits an exponential tail $\sim \exp \left[-\pi^{2}\right.$ $\left.\times D(L) t / L^{2}\right]$ in the long-time $t \rightarrow \infty$ limit. Since the distance $x$ traversed by the photon in this nonclassical random walk scales as $x \sim t^{1 / 3}$, the optical-absorption coefficient in the presence of a small imaginary part $\epsilon_{2}$ to the dielectric constant likewise scales as $\epsilon_{2}^{1 / 3}$.

In summary, it has been shown that strong localization of photons may occur in a highly predictable manner in a frequency window in certain disordered superlattice microstructures of sufficiently high dielectric contrast. These materials are the photonic analog of amorphous semiconductors, in which the interplay between order and disorder leads to the restriction of coherent back- scattering to certain Bragg resonance channels. These considerations should provide guidance in the experimental search ${ }^{25}$ for photon localization. Moreover, the utilization of localization as a trigger mechanism for nonlinear or bistable response, ${ }^{26}$ as in the Kerr electro-optic effect, ${ }^{19}$ may lead to a number of useful device applications.

This work was supported in part by the National Science Foundation Grant No. DMR-85-18163.

${ }^{1}$ P. W. Anderson, Phys. Rev. 109, 1492 (1958).

${ }^{2}$ N. F. Mott, Adv. Phys. 16, 49 (1967), and Philos. Mag. 17, 1259 (1968).

${ }^{3}$ E. Abrahams, P. W. Anderson, D. C. Licciardello, and T. V. Ramakrishnan, Phys. Rev. Lett. 42, 673 (1979).

${ }^{4}$ S. John, Phys. Rev. Lett. 53, 2169 (1984).

${ }^{5}$ P. W. Anderson, Philos. Mag. B 52, 505 (1985).

${ }^{6}$ K. Arya, Z. B. Su, and J. L. Birman, Phys. Rev. Lett. 57, 2725 (1986).

${ }^{7}$ P. Sheng and Z. Zhang, Phys. Rev. Lett. 57, 1879 (1986).

${ }^{8}$ C. A. Condat and T. R. Kirkpatrick, Phys. Rev. Lett. 58, 226 (1987).

${ }^{9}$ Y. Kuga and A. Ishimaru, J. Opt. Soc. Am. A 1, 831 (1984).

${ }^{10}$ M. P. van Albada and A. Lagendijk, Phys. Rev. Lett. 55, 2692 (1985).

${ }^{11}$ P. E. Wolf and G. Maret, Phys. Rev. Lett. 55, 2696 (1985).

${ }^{12} \mathrm{~S}$. Etemad, R. Thomson, and M. J. Andrejco, Phys. Rev. Lett. 57, 575 (1986).

${ }^{13}$ E. Akkermans, P. E. Wolf, and R. Maynard, Phys. Rev. Lett. 56, 1471 (1986).

${ }^{14}$ M. J. Stephen and G. Cwilich, Phys. Rev. B 34, 7564 (1986)

${ }^{15} \mathrm{~F}$. MacKintosh and S. John, to be published.

${ }^{16}$ A. F. Ioffe and A. R. Regal, Prog. Semicond. 4, 237 (1960).

${ }^{17}$ A. Z. Genack, Phys. Rev. Lett. 58, 2043 (1987).

18 J. M. Ziman, Models of Disorder (Cambridge Univ. Press, Cambridge, 1979).

${ }^{19} \mathrm{~A}$. Yariv and P. Yeh, Optical Waves in Crystals (Wiley, New York, 1984).

${ }^{20}$ E. Yablonovitch, Phys. Rev. Lett. 58, 2059 (1987).

${ }^{21}$ S. John, Phys. Rev. B 31, 304 (1985).

${ }^{22}$ N. Ashcroft and D. Mermin, Solid State Physics (Holt, Rinehart, and Winston, New York, 1976).

${ }^{23}$ S. John and M. J. Stephen, Phys. Rev. B 28, 6358 (1983).

${ }^{24}$ S. John, C. Soukoulis, M. H. Cohen, and E. N. Economou, Phys. Rev. Lett. 57, 1777 (1986).

${ }^{25}$ G. Watson, P. Fleury, and S. McCall, Phys. Rev. Lett. 58, 945 (1987).

${ }^{26}$ W. Chen and D. L. Mills, Phys. Rev. Lett. 58, 160 (1987). 